\title{
Evaluation of acute/late toxicity and local recurrence in T1-T2 glottic carcinoma treated with accelerated hypofractionated 3D-conformal external beam radiotherapy (3D-CRT)
}

\author{
Vassilis E. Kouloulias ${ }^{1}$, Anna Zygogianni², Eftychia Mosa' ${ }^{1}$ Kalliopi Platoni ${ }^{1}$, \\ John Georgakopoulos ${ }^{1}$, Christos Antypas ${ }^{2}$, Ivelina Beli ${ }^{1}$, Maria Tolia², Paulos Maragoudakis ${ }^{3}$, \\ Ioannis Giotakis ${ }^{3}$, Zisis Papas ${ }^{4}$, Amanda Psyrri", Nikolaos Kelekis ${ }^{1}$, John Kouvaris²

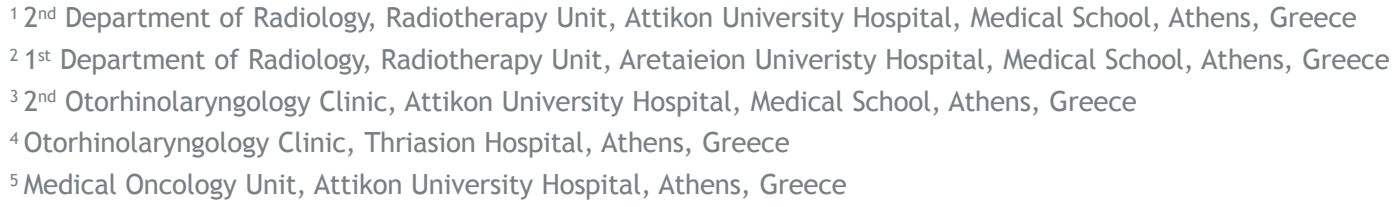

Correspondence to: Assist. Prof. Dr. Vassilis E. Kouloulias, M.D., Ph.D., $2^{\text {nd }}$ Department of Radiology, Radiotherapy Unit, ATTIKON University Hospital, Haidari, Greece. E-mail: vkouloul@ece.ntua.gr

Disclosure: No potential conflicts of interest were disclosed.

Background. The aim of the study was to evaluate the efficacy, as well as the acute and late toxicity of an accelerated hypofractionated 3DCRT schedule as radical treatment in patients with organ confined glottic cancer CT1-2N0. Patients and methods. Between June of 2004 and September 2010, 47 retrospectively selected patients (29 males, 18 females) diagnosed with organ confined $\mathrm{T} 1$ or T2 glottic cancer, were treated with external 3DCRT in an accelerated hypofractionation schedule. The median age was 70 years. A dose of $64.4 \mathrm{~Gy}$ in 28 daily fractions was prescribed. The primary study endpoints were to assess the acute and late effects of radiation toxicity, according to the EORTC/ RTOG scale, as well as the therapeutic impact of this schedule in terms of local recurrence.

Results. The median follow up was 36 months. At the end of radiotherapy, grade I, II and III acute toxicity was observed in 34, 9 and4 patients, respectively. Late grade I and II toxicity was observed in 25 and in 8 patients respectively. Only two local recurrences were observed, 15 and 24 months post 3DCRT respectively.

Conclusions. Our radiotherapy schedule achieves a high locoregional control rate with the advantage of voice preservation. The proposed hypofractionated schedule can be recommended as a standard radiotherapy treatment, since these results are comparable with those of conventional fractionation schedules.

Key words: early glottic cancer; accelerated hypofractionated radiotherapy; toxicity; local recurrence

\section{Introduction}

Cancer of the larynx is the most common cancer of the head and neck region. Risk factors include tobacco, alcohol, betel and areca nuts and deficiencies of iron, vitamin B12 and vitamin C. ${ }^{1}$

Early stage (T1 or T2) glottic cancer can be successfully treated with either radical radiotherapy or laser surgery. ${ }^{2}$ Possible treatment options in T1N0M0 disease include external beam radical radiotherapy, cordectomy, endoscopic resection (with or without laser) and partial laryngectomy. ${ }^{3}$ In T2N0M0 glottic cancer, definitive radiotherapy remains a valid treatment option with the benefit of voice preservation. ${ }^{4}$ It is usually preferred to other treatment modalities because of the high 
cure rates and the associated less impairment of vocal function. ${ }^{5}$ Surgery is usually employed after radiation as the salvage treatment in the event of locoregional relapse. Taking into account also, that a shorter hypofractionated schedule for the treatment of localized glottic cancer, will allow some sparing in radiotherapy resources in general, accelerated hypofractionated schemes are a good alternative to conventional radiotherapy fractionation. ${ }^{6}$

In our University we have established the 2.3 Gy per fraction as a standard treatment from 1997 when we also initially reported on the efficacy of accelerated hypofractionated radiotherapy for glottic carcinoma. ${ }^{7}$ With this current study we are reporting our experience in terms of the treatment outcome (response and toxicity).

\section{Patients and methods}

\section{Patients' characteristics}

Between June of 2004 and September 2010, forty-seven patients with early stage larynx cancer $(\mathrm{T} 1 \mathrm{~N} 0=38 ; \mathrm{T} 2 \mathrm{~N} 0=9$ ) were retrospectively entered to this study. Twenty nine patients were males and eighteen were females. The median age at the time of the diagnosis was 70 years (range: 57-81). All patients had a good performance status according to Eastern Cooperative Oncology Group performance score of $0-1$. The patients' characteristics are summarized in Table 1.

The pretreatment evaluation at presentation included pathology review, laryngoscopy, biopsy of the tumour, laboratory studies with complete blood count, chemistries and radiological imaging of CT and/ or MRI of the head and neck to confirm the disease stage. Eligible patients had histologically confirmed localized glottic cancer with clinical stage (cT1-2 N0) (according to American Joint Committee on Cancer staging manual, $7^{\text {th }}$ edition, 2010) and the histologic type of the malignancy was that of an invasive squamous cell carcinoma. Patients were excluded if they had a history of previous radiotherapy in head and neck region, if they were suffering from a concurrent secondary carcinoma and if they were treated for both cancers simultaneously.

The patients were referred either to ATTIKO University Hospital or to Aretaieion University Hospital of Athens in order to be treated with radical radiotherapy as the initial treatment. None of the patients received chemotherapy. All candidates had to sign an informed consent form, concerning the side effects of the hypofractionated irradiation
TABLE 1. Patients Characteristics ( $n=47)$

\begin{tabular}{ll}
\hline Parameter & Patients \\
\hline Age & \\
Median & 70 \\
Range & $57-81$ \\
Sex & \\
Male / female & $29 / 18$ \\
Tumor & \\
T1 & 38 \\
T2 & 9 \\
\hline
\end{tabular}

schedule. The primary study endpoints were to assess the acute and late effects of radiation toxicity, according to the EORTC/RTOG scale, as well as the therapeutic impact of this schedule in terms of local recurrence.

\section{Radiotherapy treatment and radiobiological assessments}

CT-scan images ( $3 \mathrm{~mm}$ slice thickness) were acquired and transferred to the treatment planning system. Patients were treated in the supine position with neck extended while immobilized with a thermoplastic head mask. Patients were instructed not to move or swallow during CT scan and simulation, and during the whole course of treatment. The CT datasets were transferred either to the Prosoma ${ }^{\circledR}$ Virtual simulation or to Plato $\AA$ contouring system, through a DICOM III network. All contouring of clinical target volume (CTV), planning target volume (PTV) and normal structures (organs at risk-OARs) was performed according to the International Commission on Radiation Units and Measurements (ICRU) criteria.

Dose calculations were performed using either the treatment planning system Eclipse (Varian Associates, Palo Alto, CA) or the PLATO (Nucletron, The Netherlands), to deliver the prescribed dose to the International Commission on Radiation Units and Measurements (ICRU) reference point.

We kept the dose range between $95 \%$ and $107 \%$ of prescribed dose. Wedge compensation was used to ensure a uniform dose distribution throughout the target volume. To evaluate the dose constraints for normal tissues we used the QUANTEC trial corrected for hypofractionation. ${ }^{8}$ 
We used linear-quadratic (LQ) modeling in order to equate the hypofractionation schedules to the Normalized Total Dose (NTD) if delivered in 2 Gy-fractions, while we also included the impact of time:-11 $B E D=n d\left(1+\frac{d}{\alpha / \beta}\right)-0.693 \frac{T-T k}{\alpha T p o t}$

where $\mathrm{n}=$ number of fractions; $\mathrm{d}=$ dose per fractions; $\mathrm{T}=$ total irradiation time in days; $\mathrm{Tk}=$ time when repopulation starts; Tpot $=$ potential doubling time. Specific values for head and neck tumours: $\alpha / \beta=10$; the $\mathrm{Tk}=21$ days; $\alpha=0.35 \mathrm{~Gy}^{-1}$ and the Tpot $=5$.

The equivalent dose by means of NTD to conventional schedule ( 35 × 2 Gy) is:

$N T D_{\text {tumor }}=\frac{\text { Dnew }\left(\frac{\alpha}{\beta}+\text { dnew }\right)+\frac{\alpha}{\beta} 0.396(\text { Teq }-21)-\frac{\alpha}{\beta} 0.396(\text { Tnew }-21)}{\frac{\alpha}{\beta}+2}$

where Dnew $=64.4 \mathrm{~Gy} ; \alpha / \beta=10 ;$ dnew $=2.3 \mathrm{~Gy} ;$ Teq $=46$ days (conventional scheme) and Tnew $=38$ days (accelerated scheme).

Thus, NTD represents the dose given in 2 Gy fractions that would give the equivalent biologic effect to the new hypofractionated dose, with a value of $\mathrm{NTD}_{\text {tumour }}=68.65 \mathrm{~Gy}$.

In case of calculations for late effect on normal tissues (OAR):

$N T D_{\text {OAR }}=D_{\text {new }}^{\text {OAR }} \frac{d_{\text {new }}^{\text {OAR }}+\alpha / \beta}{2+\alpha / \beta}$

where $D_{\text {new }}^{\text {OAR }}$ and $d_{\text {new }}^{\text {OAR }}$ are the total dose and dose per fraction, respectively, for the suggested hypofractionation scheme related to OARs, while the $\alpha / \beta$ for late effects regarding OARS was set to $\alpha / \beta=3 .{ }^{10,11}$ Under the above conditions, $\mathrm{NTD}_{\mathrm{OAR}}=68.26 \mathrm{~Gy}$.

The patients were treated with lateral opposed fields. The margins of these fields were set according to CTV and PTV delineation, usually with the superior border at the level of hyoid bone and the inferior border set at the inferior margin of the cricoid cartilage.

Weighted beams and wedges were used as necessary, to improve dose homogeneity. In general, the fields were placed isocentrically. Radiotherapy was delivered once daily 2.3 Gy per fraction, five times a week for a period of 28 days. For the treatment technique, histograms were generated and a number of parameters, including mean, median and maximum dose, were evaluated. Patient setup was monitored weekly using portal films.

Patients were treated with megavoltage equipment, either on a VARIAN CLINAC 600C Linac with $6 \mathrm{MV}$ photons, or ELECTA 6MV Linac. Portal films with amorphous siliceous electronic image device were obtained in the treatment position with therapeutic beam to confirm adequate coverage. ${ }^{12}$

\section{Follow up}

The patients were examined weekly during the treatment by indirect laryngoscopy and reviewed every month later on, after the radiotherapy, in order to assess acute/ late toxicities.
Symptoms occurring in the intervals between the start of radiotherapy and 90 days after this time point are classified as "acute". "Late" radiation complications were defined as those appearing 3 months from the end of treatment. The evaluation of acute and late radiation induced toxicity was done with the EORTC/RTOG toxicity criteria. Median follow-up duration was 36 months (range: 15-96 months).

\section{Statistical analysis}

The overall survival rate (OS) and local recurrence free survival (RFS) rates were calculated from the onset of the radiotherapy, using Kaplan-Meier method. The analysis was performed with the SPSS ver 10 software (IL, USA).

Event for OS was death related to the disease. Failure after radiotherapy was considered an event when calculating the RFS. The surgical control after radiotherapy was not considered in this study.

\section{Results}

Nearly all patients completed the planned 3D-CRT. Forty five patients completed the irradiation schedule with $64.4 \mathrm{~Gy}$ in 28 daily fractions, while two patients failed to receive the whole treatment course 
with 28 fractions. These two patients received 26 fractions with 2.3 Gy per fraction, while they didn't complete the scheme due to moderate acute effects (severe edema in the larynx).

\section{Assessment of tolerability and acute treatment-related toxicity}

Forty three patients developed mucosal reactions during the treatment (mainly arytenoid oedema) that necessitated the treatment with non-steroidal drugs and corticosteroids. At the end of radiotherapy, grade I, II and III acute skin toxicity was observed in 34, in 9 and in 4 patients, respectively. There were no patients with severe (grade 4) reported toxicities. Late toxicity as grade I, II was observed in 25 and in 8 patients respectively. No patient experienced a severe late radiation reaction (grade III or more), like laryngeal edema that required tracheotomy after the completion of radiotherapy. In details, the acute and late toxicity score is shown in Table 2.

The OS was $97.8 \%$ at 3 years. Figure 1 shows the Kaplan-Meier curve of OS and RFS, during followup. Only two local recurrences were observed 15 and 24 months post 3DCRT, respectively. Of the two patients that failed to complete the treatment, the first experienced relapse 15 months after radiotherapy and underwent surgical excision. $\mathrm{He}$ experienced a second relapse with cervical nodal metastatic disease, so he received radiotherapy, via intensity modulated radiotherapy (IMRT), but, unfortunately, he succumbed to his disease six months after re-irradiation. ${ }^{13}$ The second patient had a recurrence 24 months after radiotherapy and recently he completed the re-irradiation schedule, via IMRT. The RFS in general was $95.7 \%$ at 3 years.

\section{Discussion}

Laryngeal cancer is the most common cancer of the head and neck region. Radiotherapy is an effective treatment for early laryngeal cancer with the advantage of larynx preservation. Reported 5-year control rates are $85-95 \%$ for T1N0 disease and $75-$ $85 \%$ for T2N0 disease. ${ }^{14}$ Our study showed that RFS was $95.7 \%$ at 3 years and the overall survival was $97.8 \%$ at 3 years.

The acute toxicity reported was Grade I-II and in terms of mild hoarseness, erythema of the mucosa and less sore throat and cough requiring antitussive medication. The incidence of moderate and severe toxicity increased during the treatment,

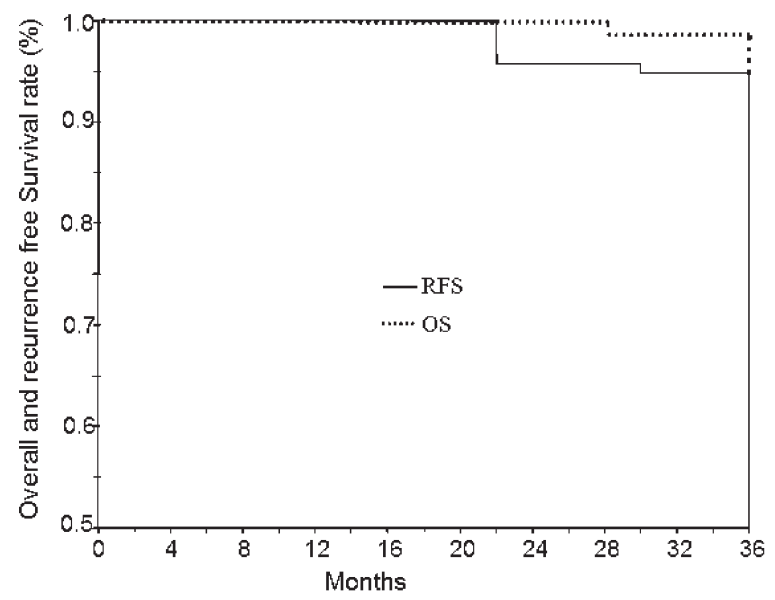

FIGURE 1. Recurrence free survival (RFS) and overall survival (OS) in patients with T1-2 glottic carcinoma.

with a peak at the 6th week or irradiation, and then progressively decreased up to 3 months after the end of radiotherapy. The late toxicity observed was mainly hoarseness and arytenoids edema, but none of our patients experienced grade III-IV toxicity.

A number of prognostic factors like the $\mathrm{T}$ stage and impaired cord mobility have been well documented, while according to a number of recent studies, larger fraction sizes and shorter overall treatment times have led to improved outcomes. More specifically, the fraction size has proved to be the only significant predictor for the laryngectomy free survival (LFS). ${ }^{15,16}$

Some investigators have reported that a long overall treatment time in cases of T1-T2 glottic cancers, has a negative impact on the treatment outcome. Skladowski et al. reported that a 10-day prolongation of overall treatment time, from 45 days to 55 days, decreased the tumour control probability by $13 \% \cdot{ }^{17,18}$ Onimaru et al. ${ }^{19}$ reported that overall treatment time plays an important role in the clinical outcome for the glottic cancer treatment. The decrease of the treatment time proved to be useful for overcoming tumour repopulation and achieving a good local control for tumours that have a high $a / \beta$ ratio and short potential doubling time. A large dose per fraction appeared to be beneficial because of the short overall treatment time, not because of any direct effects exerted by the large dose per fraction. The results of our study are in accordance with the relative results demonstrated from the study of Onimaru et al. More specifically, our report demonstrated that large doses per fraction and shorter overall treatment time succeeded a $97.8 \%$ survival rate and showed that RFS was $95.7 \%$ after a three years' follow-up. 
TABLE 2. Acute and late radiation induced skin toxicity according to EORTC/RTOG criteria

\begin{tabular}{|c|c|c|c|}
\hline & & Patients & Percentage \\
\hline \multicolumn{4}{|c|}{ Acute toxicity } \\
\hline Grade 0 & None & 0 & \\
\hline Grade 1 & $\begin{array}{l}\text { Mild or intermittent hoarseness } \\
\text { Cough not requiring antitussive } \\
\text { Erythema of mucosa }\end{array}$ & $34 / 47$ & $72.3 \%$ \\
\hline Grade 2 & $\begin{array}{l}\text { Persistent hoarseness but able to vocalize } \\
\text { Referred ear pain, sore throat, patchy } \\
\text { fibrinous exudate or mild arytenoid edema } \\
\text { not requiring narcotic } \\
\text { Cough requiring antitussive }\end{array}$ & $9 / 47$ & $19.1 \%$ \\
\hline Grade 3 & $\begin{array}{l}\text { Whispered speech, throat pain or referred } \\
\text { ear pain requiring narcotic } \\
\text { Confluent fibrinous exudate, marked } \\
\text { arytenoid edema }\end{array}$ & $4 / 47$ & $8.5 \%$ \\
\hline Grade 4 & $\begin{array}{l}\text { Marked dyspnea, stridor or hemoptysis with } \\
\text { tracheostomy or intubation necessary }\end{array}$ & 0 & \\
\hline \multicolumn{4}{|c|}{ Late Toxicity } \\
\hline Grade 0 & None & $14 / 47$ & $29.8 \%$ \\
\hline Grade 1 & $\begin{array}{l}\text { Hoarseness } \\
\text { Slight arytenoid oedema }\end{array}$ & $25 / 47$ & $53.2 \%$ \\
\hline Grade 2 & $\begin{array}{l}\text { Moderate arytenoids oedema } \\
\text { Chondritis }\end{array}$ & $8 / 47$ & $17 \%$ \\
\hline Grade 3 & $\begin{array}{l}\text { Severe oedema } \\
\text { Severe chondritis }\end{array}$ & 0 & 0 \\
\hline Grade 4 & Necrosis & 0 & 0 \\
\hline
\end{tabular}

According to Short et al. ${ }^{15}$, actuarial local control, overall survival and LFS were assessed using Kaplan-Meier method and were measured from the date of starting radiotherapy to the date of the last follow-up or death. Potential predictors of outcome of death, disease relapse and laryngectomy were assessed by univariate and multivariate analyses. These predictors included sex, anterior commissure involvement, dose per fraction $(\leq 2.25$ vs. $>2.25 \mathrm{~Gy})$, field size $\left(<25\right.$ vs. $\left.\geq 25 \mathrm{~cm}^{2}\right)$, overall treatment time ( $\leq 30$ vs. $>30$ days) and tumour differentiation (well vs. poorly differentiated). The most significant prognostic factors for local control resulted to be the extent of the disease, the fraction size and the overall treatment time.

Apart from that, Duncan et al. ${ }^{20}$ reported a review, assessing the importance of treatment gaps on the outcome of radiotherapy for laryngeal cancer. A significance increase in local relapse rates was noted if treatment time exceeded 31 days and an increase in laryngeal cancer deaths if treatment time exceeded 30 days.

Additionally, Le et al..$^{21}$ carried out a retrospective analysis of over 400 patients with T1 and T2 glottic cancer, assessing 15 potential prognostic factors influencing local control. On multivariate analysis, fraction size had a significant effect on lo- cal control in T2 group along with total dose, overall treatment time, treatment era, cord mobility and subglottic extension. Fraction size and overall treatment time were significant in T1 disease on the univariate analysis only.

Yu et al. ${ }^{22}$ assessed the effect of fraction size in a large retrospective review of patients with T1 glottic cancer treated with differing dose per fraction over a 10-year period, 1978-1988. Patients treated with $>2$ Gy per fraction, had an improvement in local control at $84 \%$, compared with $65.6 \%$ for 2 Gy per fraction at 84-month follow-up. Fraction size was the only significant predictor of the outcome on univariate and multivariate analyses.

Gowda et al. ${ }^{23}$ reviewed 200 patients with T1 glottic cancer from the Christie and Royal Marsden Hospitals treated with a 3-week regimen delivering 50-52.5 Gy in 16 fractions. Their report mentioned an excellent 5-year local control rate (93\%) and cause-specific survival (97\%) with minimal toxicity. Furthermore, the study reported that overall treatment time and large fraction size may be advantageous in this group of patients with laryngeal cancer.

Hypofractionated radiotherapy in general has had great appeal as a strategy for improving the therapeutic gain of patients treated with radio- 
therapy and especially for patients with head and neck cancer. In line with these studies, our report also demonstrated the efficacy and safety of hypofractionated radiotherapy in early glottic cancer. At the same time, we showed that a 3DCRT hypofractionated schedule up to $64.4 \mathrm{~Gy}$, with a 2.3 Gy daily fraction, not only managed to succeed high proportion rates of overall survival $(97.8 \%)$, RFS (95.7\%) and preservation of the voice, but also gave the opportunity of a treatment to patients that are unable to visit the hospital for a long period of time. Additionally, hypofractionated radical radiotherapy of early glottic cancer may be beneficial for the logistics of a hospital, succeeding a shorter radical treatment schedule and consequently a shorter patients' waiting list.

Agarwal et al..$^{24}$ reported that hypofractionation did not show any negative impact and that there is a considerable improvement of voice quality following radiotherapy. According to Di Nicola et al. ${ }^{25}$ in their institution study, 40 patients with T1 squamous cell carcinoma of the true vocal cord were irradiated with curative intent. The results of the report showed that although all patients before radiotherapy had an overall voice quality deteriorating, the vocal performance was strongly improved at 36 months after irradiation. Thus, there was a significant difference between pre- and post-radiotherapy for both groups.

The studies of Cheah et al. and Kazi et al., enhance the result that hypofractionation for T1NOM0 larynx carcinoma offers high locoregional control rates with voice preservation. ${ }^{6,26}$

\section{Conclusions}

External radiotherapy is an effective treatment for early glottic carcinomas. Our retrospective study demonstrated that 3D-CRT hypofractionation up to $64.4 \mathrm{~Gy}$ in 28 daily fractions, is a feasible and safe modality, achieving improved local control rates, overall survival, laryngectomy free survival, decreased levels of toxicity, while at the same time preserves and improves the quality of voice. At last but not least, it seems that the accelerated schedule of 3DCRT with 2.3 Gy per fraction is a good alternative for radiotherapy departments with high workload. However, a randomized study is needed to confirm our results: due to the retrospective nature of our study, the small number of evaluated patients, and selection of patients included should be considered as preliminary.

\section{References}

1. Zygogianni A, Kyrgias G, Mystakidou K, Antypas C, Kouvaris J, Papadimitriou $C$, et al. Potential role of the alcohol and smoking in the squamous cell carcinoma of the head and neck: review of the current literature and new perspectives. Asian Pac J Cancer Prev 2011; 12: 339-44.

2. Krengli M, Policarpo M, Gambaro G, Panella M, Manfredda I, Alyffa P, et al. Voice quality after treatment for T1a glottic carcinoma - radiotherapy versus laser coredctomy. Acta Oncol 2004; 43: 284-9.

3. Goor KM, Peeters AJ, Mahieu HF, Langendijk JA, Leemans CR, Verdonck-de Leeuw IM, et al. Cordectomy by CO2 laser or radiotherapy for small T1a glottic carcinomas: costs, local control, survival, quality of life and voice quality. Head Neck 2007; 29: 128-36.

4. Loughran S, Calder N, MacGregor FB, Carding P, MacKenzie K. Quality of life and voice following endoscopic resection or radiotherapy for early glottis cancer. Clin Otolaryngol 2005; 30: 42-7.

5. Rhys-Evans P, Montgomery PQ, Gullane PJ. Principles and practice of head and neck oncology. London: Martin Dunitz; 2003.

6. Kazi $R$, Venkitaran $R$, Johnson $C$, Prasad $V$, Clarke $P$, Newbold $K$, et al. Prospective, longitudinal electroglottographic study of voice recovery following accelerated hypofractionated radiotherapy for $\mathrm{T} 1 / \mathrm{T} 2$ larynx cancer. Radiother Oncol 2008; 87: 230-6.

7. Plataniotis G, Kouvaris J, Hatzimichalis H, Papavasiliou CG, Vlachos L. Results of radiotherapy for $\mathrm{T} 1$ carcinoma of the glottis by fractions of $2.3 \mathrm{~Gy}$ per day. Acta Oncol 1997; 36; 342-3.

8. Marks LB, Yorke ED, Jackson A, Ten Haken RK, Constine LS, Eisbruch A, et al. Use of normal tissue complication probability models in the clinic. Int Radiat Oncol Biol Phys 2010; 76(3 Suppl): S10-9.

9. Fowler J. The linear quadratic formula and progress in fractionated radiotherapy. Br J Radiol 1989; 62: 679-94.

10. Emami B, Lyman J, Brown A, Coia L, Goitein M, Munzenrider JE, et al. Tolerance of normal tissue to therapeutic irradiation. Int J Radiat Oncol Biol Phys 1991; 21: 109-22.

11. Thames HD, Bentzen SM, Turesson I, Overgaard M, Van den Bogaert W. Time-dose factors in radiotherapy: a review of the human data. Radiother Oncol 1990; 19: 219-35.

12. Pesznyák C, Polgár I, Weisz C, Király R, Zaránd P. Verification of quality parameters for portal images in radiotherapy. Radiol Oncol 2011; 45: 68-74.

13. Zygogianni A, Kyrgias G, Kouvaris J, Antypas C, Theodorou K, Papageorgiou $\mathrm{S}$, et al. Re-irradiation in head and neck cases using IMRT technique: a retrospective study with toxicity and survival report. Head Neck Oncol 2012; 4: 78.

14. Gowda RV, Henk JM, Mais KL, Sykes AJ, Swindell R, Slevin NJ. Three weeks radiotherapy for T1 glottic cancer: the Christie and Royal Marsden Hospital experience. Radiother Oncol 2003; 68: 105-11.

15. Short S, Krawitz H, Macann A, West T, Morton RP, Mclvor NP, et al. T1NO/ T2N0 glottic carcinoma: a comparison of two fractionation schedules. Austr Rad 2006; 50: 152-7.

16. Dinshaw KA, Sharma V, Agarwal JP, Ghosh S, Havaldar R. Radiation therapy in T1-T2 glottic carcinoma: influence of various treatment parameters on local control/ complications. Int J Radiat Oncol Biol Phys 2000; 48: 723-35.

17. Parsons JT, Greene BD, Speer TW, Kirkpatrick SA, Barhorst DB, Yanckowitz T. Treatment of early and moderately advanced vocal cord carcinoma with 6MV x-rays. Int J Radiat Oncol Biol Phys 2001; 50: 953-9.

18. Skladowski K, Tarnawski R, Maciejewski B, Wygoda A, Slosarek K. Clinical radiobiology of glottis T1 squamous cell carcinoma. Int J Radiat Oncol Biol Phys 1999; 43: 101-6.

19. Onimaru R, Hasegawa M, Yasuda K, Homma A, Oridate N, Fukuda S, et al. Radiotherapy for glottic T1NO carcinoma with slight hypofractionation and standard overall treatment time: Importance of overall treatment time. Jpn J Clin Oncol 2011; 41: 103-19.

20. Duncan W, MacDougall RH, Kerr GR, Downing D. Adverse effect of treatment gaps in the outcome of radiotherapy for laryngeal cancer. Radiother Oncol 1996; 41: 203-7. 
21. Le QT, Fu KK, Kroll S, Ryu JK, Quivey JM, Meyler TS, et al. Influence of fraction size, total dose and overall time on local control of T1-T2 glottic cancinoma. Int J Radiat Oncol Biol Phys 1997; 39: 115-26.

22. Yu E, Shenouda G, Beaudet MP. Impact of radiation therapy fraction size on local control of early glottis carcinoma. Int J Radiat Oncol Biol Phys 1997; 37: 587-91.

23. Gowda RV, Henk JM, Mais KL, Sykes AJ, Swindell R, Slevin NJ. Three weeks radiotherapy for T1 glottic cancer: the Christie and Royal Marsden Hospital expperience. Radiother Oncol 2003; 68: 105-11.

24. Agarwal J, Baccher GK, Waghmare CM, Mallick I, Ghosh-Laskar S, Budrukkar $A$, et al. Factors affecting the quality of voice in the early glottis cancer treated with radiotherapy. Radiother Oncol 2009; 90: 177-82.

25. Di Nicola L, Gravina GL, Marampon F, Bonfili P, Buonopane S, Di Staso M, et al. The impact of conventional or hypofractionated radiotherapy on voice quality and oncological outcome in patients with early glottic cancer. Oncol Reports 2010; 24: 1383-8.

26. Cheah NL, Lupton S, Marshall A, Hartley A, Glaholm J. Outcome of the T1NOMO squamous cell carcinoma of the larynx treated with short-course radiotherapy to a total dose of $50 \mathrm{~Gy}$ in 16 fractions: the Birmingham Experience. Clin Oncol 2009; 21: 494-501. 